MS01-05

\section{Serial micro- and nano-crystallography at the European XFEL: Capabilities of and first results from the $\mathrm{SPB} / \mathrm{SFX}$ instrument}

Stephan Stern

1. European XFEL, Hamburg, Germany

email: stephan.stern@xfel.eu

The rapid evolution of micro- and nanocrystallography as an important and distinct field of macromolecular crystallography was, from its very beginning, closely tied to the advent of ultrafast, pulsed X-ray sources of the 4th generation such as X-ray free-electron lasers (XFELs). Today, serial femtosecond nanocrystallography experiments at XFELs have proven to be a powerful method for time resolved studies of fundamental biological dynamics on the molecular level, ranging from enzyme-substrate mixing to photo-induced timed resolved dynamical processes. For the new X-ray free-electron laser facility European XFEL, dynamical biology in the sense of time resolved micro- and nanocrystallography was determined to be one of the major pillars of scientific research, and the envisaged X-ray pulse repetition rates in the Megahertz regime at European XFEL promise to offer unique capabilities for data collection for such experiments.

Here, we present capabilities, experiences and results from the first run of experiments at the SPB/SFX scientific instrument of the European XFEL. We give an overview of the general design and capabilities of the instrument and outline what benefits and challenges come from the uniquely high X-ray repetition rate. This includes the consequences for sample delivery, detector calibration, data collection and experimental performance in general. We further outline the capabilities for ultrafast time resolved studies at SPB/SFX aided by an optical laser system that follows the European XFEL's MHz pulse structure. Finally, plans for the incorporation and commissioning of new X-ray focussing mirror optics, and the installation of two additional experimental interaction regions at $\mathrm{SPB} / \mathrm{SFX}$ will be presented and are envisaged to greatly enhance the experimental capabilities of the instrument for micro- and nanocrystallography.References:

Keywords: XFEL, instrument, time-resolved
MS02 - From data collection to structure finalization: getting the most from your crystal Chairs: Dr. William Shepard, Dr. Katherine McAuley

MS02-01

\section{Achieving higher performance in high- throughput compound and fragment screening campaigns by using "Club Class" data collection, Pipedream and CRIMS}

Gerard Bricogne ${ }^{1}$, Gerard Bricogne ${ }^{1}$, Claus Flensburg ${ }^{1}$, Peter Keller $^{1}$, Wlodek Paciorek ${ }^{1}$, Andrew Sharff ${ }^{1}$, Clemens Vonrhein ${ }^{1}$, Jose Antonio Marquez ${ }^{2}$, Djordje Musil ${ }^{3}$

1. Global Phasing Ltd., Cambridge, United Kingdom

2. HTX Laboratory, EMBL Grenoble Outstation, Grenoble, France)

3. Discovery Technologies, Merck KGaA, Darmstadt, Germany)

email: gb10@globalphasing.com

Fourth-generation X-ray sources, fast sample delivery systems for micro-crystals and pixel-array detectors with ever faster frame rates are radically extending ways of collecting diffraction data, while associated software developments are creating a continuum of options between serial experiments at synchrotrons and at XFELs.

From the viewpoint of structure-based drug discovery, these developments will give better access to targets for which "macro-crystals" cannot be obtained, but they will do so at a greatly increased cost. This encourages renewed efforts to more fully exploit the intrinsic advantages of macro-crystals, when available, through better experiments. Unfortunately, strong emphasis on automation and speed in typical high-throughput data collection workflows has left little room for invoking any form of expert decision making in data collection without considerable disruption of automation.

An unlikely opportunity linked to the development of the Diamond I23 experimental phasing beamline (Armin Wagner) has enabled us to address this limitation via a "Third-party design and control" approach that makes our solution transferable between different beamline software environments with minimum effort. This approach has been validated by the live execution of our workflows on Diamond beamlines running under the GDA control software, and recently on the ESRF ID30B beamline running under $\mathrm{MXCuBE}$.

We have been able to demonstrate that "Club Class" multi-orientation datasets can significantly enhance the detection and characterisation of small weakly bound fragments - a process fundamental to fragment-based drug discovery - in the context of a fragment screening campaign. Numerous datasets collected on the ESRF MASSIF-1 beamline were processed with the Global Phasing "Pipedream" software suite that seamlessly combines automated data reduction and model refinement with ligand density elicitation, automated ligand fitting and ligand geometry 